\title{
MANEJO TERAPÊUTICO DO PACIENTE COM DISFUNÇÃO OLFATÓRIA: REVISÃO DA LITERATURA
}

\author{
THERAPEUTIC MANAGEMENT OF THE PATIENT WITH OLFACTORY \\ DYSFUNCTION: A LITERATURE REVIEW
}

Henrique de Paula Bedaque ${ }^{1}$; Juliane Patrícia Grigório da Silva ${ }^{1}$; Mônica Claudino Medeiros Honorato1; Deborah Carla Santos Gibson ${ }^{1}$; Halan Araújo Santos ${ }^{1}$; Lucas Marinho Vasconcelos ${ }^{1}$; Lidiane Maria de Brito Macedo Ferreira ${ }^{2}$

1. Residente em otorrinolaringologia pela Universidade Federal do Rio Grande do Norte (UFRN). Natal-RN.

2. Professora Adjunta de otorrinolaringologia do Departamento de Cirurgia, UFRN. Natal-RN.

Artigo produzido no Hospital Universitário Onofre Lopes (UFRN).

Financial support: None.

Conflicts of interest: None.

Endereço: Hospital Universitário Onofre Lopes, Departamento de Cirurgia. Av. Nilo Peçanha, 620, $3^{\circ}$ subsolo, Petrópolis, Natal/RN CEP:59012-300.

Submitted: aug 04; accepted after revision, nov 25, 2020.

\begin{abstract}
Objective: Olfactory dysfunction has been shown to be a very prevalent alteration in patients with COVID-19, both as a clinical presentation and as a sequel of duration not yet determined. Thus, this research seeks to understand how the new publications on the treatment of hyposmia and anosmia have brought therapeutic opportunities. Methodology: This is a literature review of articles published on the topic of olfactory dysfunction and treatment, in the MEDLINE + PUBMED database. The research was designed using the following descriptors: "hyposmia OR anosmia AND treatment". Then the filters were used: "last 05 years" and "human". In addition to the following types of study: "Clinical Trial", "Systematic Review" and "Meta-analyzes". Totaling 55 articles, of which we highlight 13 articles with consistent summary for the purpose of this review. Conclusion: Most of the recently published studies focus on treatment based on two interventions: the use of corticosteroids and olfactory training, showing good results but in fragile studies. In addition, therapeutic opportunities with the use of intranasal insulin, positive pressure devices and nasal lavage with sodium citrate have appeared in recent publications.
\end{abstract}

Key words: Olfaction Disorders. Therapeutics. Drug therapy and Smell. 


\section{RESUMO:}

Objetivo: A disfunção olfatória vem se mostrando uma alteração muito prevalente nos pacientes com COVID-19, tanto como apresentação clínica como sequela de duração ainda não determinada. Dessa forma, essa pesquisa busca entender o que as novas publicações sobre o tratamento da hiposmia e anosmia vem trazendo de oportunidades terapêuticas. Metodologia: Trata-se de uma revisão sistemática da literatura sobre artigos publicados sobre o tema disfunção olfatória e tratamento, na base de dados MEDLINE + PUBMED. A pesquisa foi delineada utilizando os seguintes descritores: "hyposmia OR anosmia AND treatment". Em seguida foram utilizados os filtros: "últimos 05 anos" e "humanos". Além dos seguintes tipos de estudo: "Ensaio Clínico", "Revisão Sistemática" e "Metanálises". Totalizando 55 artigos, dos quais destacamos 13 artigos com resumo condizente com o objetivo desta revisão. Conclusão: A maioria dos estudos recentemente publicados foca no tratamento com base em duas intervenções: o uso do corticoide e o treinamento olfatório, apresentando bons resultados, porém em estudos frágeis. Além disso, oportunidades terapeuticas com o uso da insulina intranasal, dos aparelhos de pressão positiva e da lavagem nasal com citrato de sódio vem aparecendo em publicações recentes.

Palavras-chave: Transtorno do olfato. Terapêutica. Tratamento Farmacológico e olfato.

\section{INTRODUÇÃO}

O olfato é um dos sentidos mais primitivos da história da evolução humana, nos traz a possibilidade de defesa antes mesmo que a visão consiga detectar o perigo, como no exemplo de um incêndio ou vazamento de gás numa residência ${ }^{1}$. Além disso, por meio dos perfumes naturais ou artificiais sentimos prazer com a liberação de dopamina². Assim, por esses motivos, a perda do olfato afeta de modo importante a qualidade de vida das pessoas ${ }^{3}$.

Essa perda de olfato pode ser caracterizada por diversas formas, como na hiposmia (redução parcial da capacidade de sentir cheiros) e na anosmia (perda total do olfato), que são as formas mais comuns ${ }^{3}$.

Nesse contexto, sabemos que as três principais causas de disfunção olfatória são as Infecções de vias aéreas superiores (IVAS), as Rinossinusites e o Traumatismo crânioencefálico (TCE). Em meio à Pandemia pelo COVID-19 no biênio 2019-2020, a disfunção olfativa se mostrou um sintoma que pode chegar para até $85,6 \%$ dos pacientes infectados, o que acendeu novos olhares para essa disfunção ainda pouco estudada 4 . Entretanto, em termos de tratamento, as evidências atuais pouco auxiliam em condutas eficazes, principalmente quando a fisiopatologia envolve lesões neurais e corticais do olfato ${ }^{3}$.

Dessa forma, considerando que a infecção por COVID-19 se comporta inicialmente como uma IVAS, evidências indicam que há possibilidade dessa infeç̧ão 
Manejo terapêutico do paciente com disfunção olfatória: revisão da literatura

Bedaque HP; et al

trazer tanto danos temporários, como os causados pela obstrução nasal durante o período sintomático, como perenes, em situações nas quais o vírus adentrou o nervo olfatório e trouxe lesão neural, essa entrada se da por meio dos receptores ACE2 ${ }^{5}$. Há relatos na literatura da capacidade de vírus respiratórios utilizarem o nervo olfatório como transporte para danificar zonas corticais do olfato ${ }^{3,4}$.

A presente revisão surge da expectativa de aumento no presente e no futuro do número de pessoas com sintomas de hiposmia e anosmia que irão procurar ajuda médica para manejo terapêutico. Logo, uma revisão da literatura mais recente sobre esse tema se torna de grande valia para atualizar o profissional da saúde sobre possíveis terapêuticas e prepará-lo melhor aos próximos passos da ciência.

\section{METODOLOGIA}

Trata-se de uma revisão sistemática da literatura de artigos publicados sobre o tema de tratamento de hiposmia e anosmia nas bases de dados MEDLINE e PUBMED. A pesquisa foi delineada utilizando os seguintes descritores: "hyposmia OR anosmia AND treatment". Em seguida foram selecionados os seguintes filtros: "Últimos 05 anos"; "humanos"; "inglês"; "Português" além dos seguintes tipos de estudos: "Clinical Trial"; "Meta-analysis" e "Revisão". Após os filtros, obteve-se na pesquisa realizada no dia 21 de abril de 2020 um total de 55 artigos aptos à avaliação de seus títulos e resumos.

Dessa forma, após a avaliação de dois pesquisadores dos títulos e resumos dos 55 artigos encontrados na busca, apenas 13 se encontravam realmente condizentes com o tema de tratamento de pacientes com qualquer tipo de hiposmia ou anosmia.

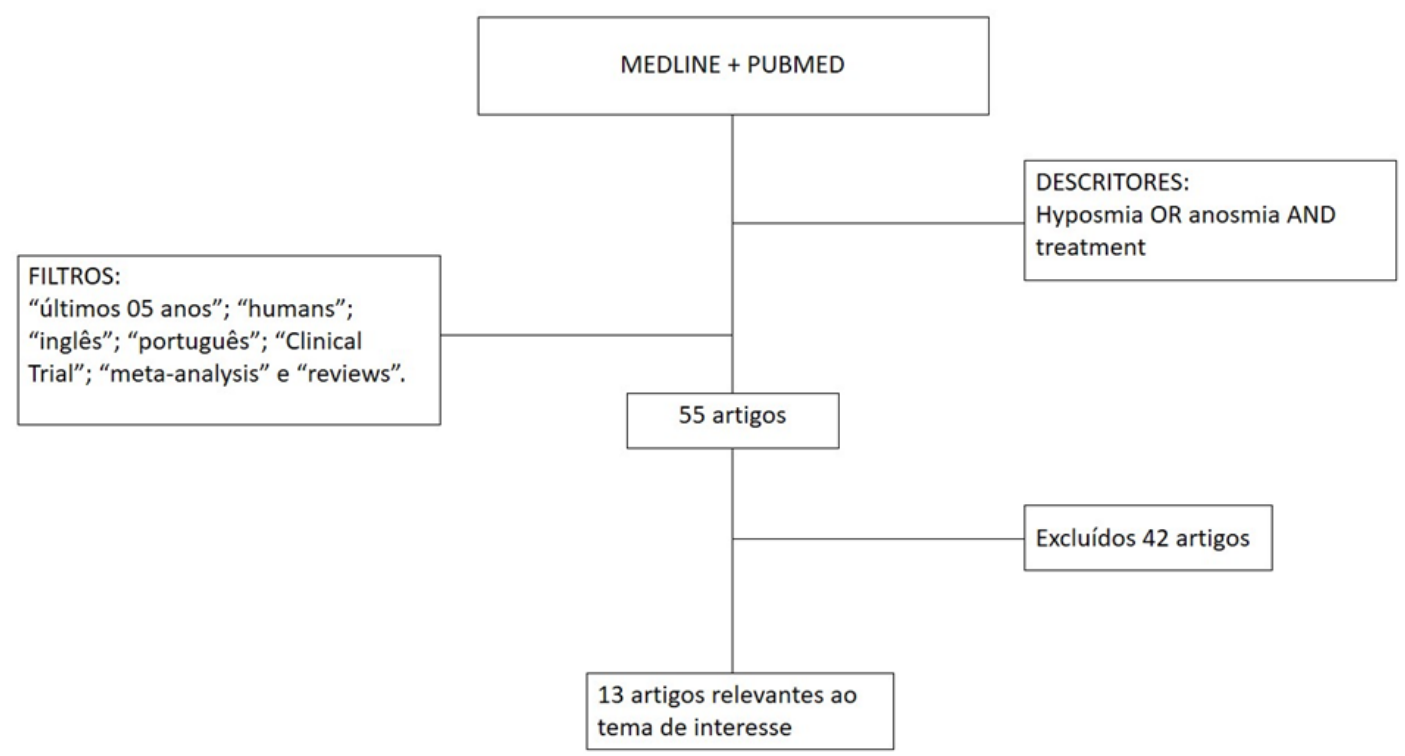

Figura 1 - Esquema do processo de seleção de artigos para revisão. 
Manejo terapêutico do paciente com disfunção olfatória: revisão da literatura Bedaque HP; et al

\section{RESULTADOS E DISCUSSÃO}

Estudos sobre o manejo terapêutico de pacientes com disfunção olfativa ainda carecem de grandes evidências ou de eficácia clínica expressiva, o que se observa é q ue existem certas condutas consagradas por alguns estudos e decisão de especialistas. Desse modo, o uso de corticoides, nasais ou sistêmicos, tem mostrado bons resultados quando o paciente tem os sintomas em decorrência de uma rinossinusite ${ }^{3,6}$.

Além disso, algumas deficiências olfativas ocorridas após um TCE podem ter indicação cirúrgica para facilitar a regeneração neural ${ }^{3}$. Assim, alguns tratamentos pontuais e específicos para algumas etiologias vêm sendo desvendadas com as últimas pesquisas científicas. Comentaremos abaixo alguns manejos.

\section{Uso de corticoide}

Um estudo ensaio clínico randomizado, não cego, procurou comparar dois grupos de pacientes com hiposmia sem evidências de rinossinusite crônica em relação à duas intervenções: treinamento olfatório e irrigação nasal com budesonida 7 . 0 primeiro grupo (67 pacientes) foi submetido a treinamento olfatório e irrigação salina (controle), e o segundo (66 pacientes) teve intervenção com treinamento olfatório e irrigação com budesonida. A recuperação olfatória foi avaliada em termos do escore University of Pennsylvania Smell Identification Test (UPSIT) ${ }^{7}$.

Observou-se recuperação em 11 pacientes (26.9\%) do controle versus 9 pacientes (43.9\%) do grupo com budesonida, com Odds Ratio bruta de 2.13 a favor do grupo intervenção (IC 95\% 1.03-4.41) ( $p=0.039$ ) e Odds Ratio ajustada de 3.93 (IC 95\% 1.20-12.88) ${ }^{7}$. Dessa forma, o estudo mostrou um potencial benefício da irrigação com corticoide associada ao treinamento olfatório para uma melhor resposta terapêutica.

Além disso, em relação a métodos de entrega da medicação à cavidade nasal, foi feito estudo, não cego, para avaliar se havia diferença nos escores de hiposmia (pelo Sniffin' Sticks) entre paciente submetidos a spray nasal convencional com dexametasona e àqueles submetidos ao denominado AMSA $^{\circledR}$ (dispositivo aerolizador, combinando pressão e vibração) ${ }^{8}$.

Constatou-se que houve melhora dos escores olfatórios em ambos os grupos após 2 semanas (médias de 2,2 pontos com $A_{M S A}{ }^{\circledR}$ e 2,6 pontos com spray nasal), porém sem diferença estatisticamente significativa entre os métodos $(p=0,82)$. Ademais, após 6 semanas de follow-up sem tratamento, houve deterioração olfatória de volta à linha de base $\left(\mathrm{AMSA}^{\circledR}\right.$ : queda de 1,8 pontos; spray nasal: queda de 1,2 pontos) $(p=0,005)^{7}$. Dessa forma, a escolha do dispositivo para administração do corticoide, seja pelo spray nasal ou pelo dispositivo, não deve alterar o resultado do tratamento ${ }^{8}$.

Outro estudo randomizado avaliando a função olfativa de 30 pacientes portadores de rinite alérgica sazonal, obedecendo aos critérios do ARIA, foram divididos em 3 grupos de 10 pacientes e acompanhados por 4 semanas. 0 grupo 1 recebeu 
Manejo terapêutico do paciente com disfunção olfatória: revisão da literatura

Bedaque HP; et al

montelucaste sódico (10mg/dia) e furoato de mometasona nasal ( $200 \mathrm{mcg} / \mathrm{dia})$, grupo 2 recebeu montelucaste sódico (10mg/dia) e o grupo 3 recebeu furoato de mometasona nasal $(200 \mathrm{mcg} / \mathrm{dia})^{9}$.

Os pacientes tiveram avaliação do olfato pelo teste olfatório Sniffn' Sticks, obtendo as médias do limiar olfatório, da capacidade de identificação, da discriminação olfativa e do TDI Score (somatória do limiar, identificação e discriminação olfativa) antes e após as 04 semanas de seguimento. Os grupos 1 e 3 apresentaram melhora estatisticamente significativa, com o TDI score passando de $31,27 \pm 3,24$ para 36,36 \pm $3,07(p=0,003)$ e de 30,59 $\pm 2,57$ para 33,91 $\pm 2,16(p=0,01)$, respectivamente. Entretanto, não houve melhora significativa para o grupo 2. Concluiu-se então que o montelucaste sódico não apresentou melhora estaticamente significativa da função olfativa e que a melhora no grupo que recebeu montelucaste sódico e furoato de mometasona deveu-se ao efeito do furoato mometasona ${ }^{9}$.

Em uma revisão da literatura avaliando a eficácia de esteróides tópicos e sistêmicos no tratamento da disfunção olfatória não relacionada a inflamação nasossinusal ou rinite, encontraram diferença significativa entre o uso de corticosteróides sistêmicos e esteróides spray nasal com melhores resultados para o uso do corticoide oral. Entretanto, as evidências que sustentam essa comparação entre a forma de administração da corticoterapia são fracas ${ }^{10}$.

As evidências que sustentam o uso dos corticosteróides nasais também são conflitantes. Em um estudo randomizado duplo-cego não encontrou diferença no uso do spray nasal comparado ao placebo ${ }^{11}$.

Dessa forma, evidências discordantes e opostas ainda são muito encontradas no que se trabalha para o uso do corticoide para o tratamento da disfunção olfatória. O que representa a necessidade de estudos bem desenhados e robustos para trazer mais evidência científica.

\section{Uso do treinamento olfatório}

A plasticidade neural do sistema olfatório é única e por isso, certas intervenções, como o treinamento olfatório, têm o potencial de promover sua recuperação através do despertar dos neurônios espefícicos deste sentido ou modulando a função neural ${ }^{3}$. Por outro lado, insultos repetidos ou graves comumente resultam na falha dessa regeneração neuronal e consequente persistência de disfunção olfativa ${ }^{12}$.

O treinamento olfatório se dá através de cheiros frequentes e/ou exposição a odores fortes. Os cheiros estimulantes típicos são representativos das principais categorias de odor: florido, frutado, aromático e resinosos. Os protocolos geralmente incluem a exposição a cada odorante 2 a 4 vezes ao dia, com diferentes durações, variando de um dia a 35 semanas $^{12}$. 
Manejo terapêutico do paciente com disfunção olfatória: revisão da literatura Bedaque HP; et al

Em uma revisão da literatura com 10 estudos, 639 pacientes, para a avaliação dos efeitos do treinamento olfatório na recuperação da sua disfunção foi possível realizar uma metanálise com apenas 3 estudos. Na análise o treinamento trouxe uma melhora do Score TDI (Limiar, Discriminação e Identificação) no grupo intervenção com diferença média de 3,77 (IC 95\% 2,28 - 5,26), porém com alta heterogenicidade, de $73 \%{ }^{12}$. Além disso, na avaliação de uma melhora funcionalmente significativa (>5,5 no TDI Score) tivemos novamente uma melhor performace no grupo do treinamento olfatório com Odds Ratio de 2,75 (IC 95\% 1,60-4,73) e baixa heterogenicidade, $0 \%{ }^{12}$.

Dentro dos componentes individuais do escore do TDI, o treinamento olfatório resultou na melhoria da discriminação (MD 1,92; IC 95\%, 1,13 a 2,71) e identificação (MD 1,61; IC 95\%, 0,55 a 2,68), mas não nos limiares olfatórios (MD - 0,01; IC95\%, -0,42 a 0,39$)$. Então, conclui-se que o treinamento pode representar uma intervenção promissora para pacientes com disfunção olfativa devido a múltiplas etiologias, embora ainda sejam necessários estudos adicionais de alta qualidade, duplo cegos e controlados por placebo para definir melhor as indicações, resultados e duração da terapia ${ }^{12}$.

Em outra revisão sistemática ${ }^{13}$ analisada encontramos em pacientes que receberam treinamento olfatório uma melhora estatisticamente significativa no escore do TDI em comparação aos pacientes controle (diferença média [MD] 3,77; IC 95\%, 2,28 a 5,26). Foi observada heterogeneidade significativa, $I^{2}$ de $73 \%$; no entanto, isso provavelmente ocorreu devido ao pequeno número de estudos com foco em múltiplas etiologias $^{13}$.

Além disso, na revisão sistemática mais recente avaliou-se 13 pesquisas com um " $n$ " para o desfecho discriminação olfatória de 1005 voluntários, para este item o tamanho do efeito calculado pelo Hedges $g$ foi de 0,83 (95\% IC 0,26 - 1,4), sustentando que a prática do treinamento olfatório apresenta um grande tamanho de efeito sobre a melhora da discriminação dos odores. Entretanto, de forma semelhante a outras revisões, a heterogeneidade se mantém como um fator importante presente ${ }^{13}$.

\section{Uso do Gluconato de Zinco}

No pensamento terapêutico da disfunção olfatória relacionado a uma realidade pós-traumática identificamos um ensaio clínico randomizado avaliando a resposta terapêutica em pacientes com anosmia pós-traumática após administração de gluconato de zinco (10mg três vezes ao dia) e/ou prednisolona $(1 \mathrm{mg} / \mathrm{kg} / \mathrm{dia}$ com posterior desmame por 2 semanas). Constatou-se que houve recuperação de 11 pacientes (28.2\%) no grupo submetido a gluconato de zinco + prednisolona (grupo 1), 9 pacientes (25.7\%) no grupo tratado somente com zinco (grupo 2), 4 (11.8\%) no grupo com apenas prednisolona (grupo 3), e 1 paciente (2.7\%) no grupo controle (grupo 4) ${ }^{14}$.

Diante disso, verificou-se superioridade dos grupos 1 e 2 em relação ao grupo controle ( $p=0.006$ e $p=0.013$, respectivamente), e foi levantado a hipótese de um 
Manejo terapêutico do paciente com disfunção olfatória: revisão da literatura

Bedaque HP; et al

possível papel do zinco na terapia de anosmia pós-traumática ${ }^{14}$. Assim, o uso de prednisolona associado ao zinco se mostra como uma possibilidade terapêutica em pacientes com disfunção relacionada a um trauma ${ }^{14}$.

\section{Uso de Caroverinas}

Uma revisão sistemática ${ }^{6}$ encontrou apenas um estudo, ECR, com o uso de caroverinas, medicação também usada para o tratamento de zumbido, e esta pesquisa mostrou benefício sobre o seu uso frente ao controle com zinco. Apesar do follow-up de apenas um mês e do risco de vieses os autores da revisão veem como promissora o uso das caroverinas ${ }^{6}$.

\section{Uso de ácido alfalipóico}

Na mesma revisão ${ }^{6}$ do tópico passado foi identificado apenas um estudo sobre o uso do ácido alfalipóico, muito usado no tratamento da neuropatia diabética, no tratamento de pacientes com disfunção olfatória de etiologia pós-viral. Esse único estudo não controlado e com follow-up médio de 4,5 meses mostrou que os pacientes que utilizaram essa substância obteram melhora do TDI Score. Apesar de não ser a metodologia ideal os autores da revisão também veem de forma promissora o uso do ácido alfalipóico ${ }^{6}$.

\section{Uso da insulina intranasal}

Um ensaio clínico prospectivo, randomizado, duplo-cego e controlado realizado 2017- 2018 investigou o tratamento com insulina intranasal em paciente com queixa de hiposmia. No estudo foram incluídos 38 pacientes, sendo randomizados em dois grupos: intervenção e placebo15.

Foi empregado para classificar os pacientes com base na intensidade da hiposmia o teste Connecticut Chemosensory Clinical Research Center (CCCRC) com avaliação apenas do limiar de butanol. De acordo com a pontuação do CCCRC, são definidos os graus de intensidade da hiposmia: normosmia ( 6 a 7 pontos), hiposmia leve ( 5 a 5,75 pontos), hiposmia moderada (4 a 4,75 pontos), hiposmia severa ( 2 a 3,75 pontos) e anosmia ( 0 a 0,175 pontos $)^{15}$.

Os pacientes foram submetidos a endoscopia endonasal com colocação na fenda palatina de espuma de geofoam com insulina (40 UI) para o grupo intervenção e espuma de geofoam embebida em solução salina para o grupo placebo. O procedimento foi realizado duas vezes por semana, durante 4 semanas, com teste de limiar de butanol para avaliação do escore CCCRC antes da intervenção e 4, 8 e 16 semanas após o tratamento ${ }^{15}$.

A pesquisa mostrou que antes da intervenção não havia diferença significativa no escore CCCRC entre os grupos $(p=0,82)$ e na intensidade da hiposmia entre os grupos $(p=0,61)$. Após 4 meses de acompanhamento da intervenção, a pontuação do CCCRC 
Manejo terapêutico do paciente com disfunção olfatória: revisão da literatura Bedaque HP; et al

do grupo intervenção foi significativamente maior em comparação com o do grupo placebo $(p=0,01)$ e houve diferença entre os dois grupos em relação à intensidade da doença $(p=0,011)^{15}$.

Portanto, os achados indicam que a insulina intranasal pode desencadear a melhoria da capacidade olfativa. Não foram encontradas diferenças significativas na glicose sérica e níveis séricos de insulina dos dois grupos após o tratamento e efeitos adversos significativos não foram evidenciados ${ }^{15}$.

\section{Uso da ventilação com pressão positiva}

Um estudo prospectivo avaliou o efeito da terapia de pressão positiva nas vias aéreas (PAP) em pacientes com síndrome da apneia obstrutiva do sono (SAOS) e comprometimento olfatório ${ }^{16}$.

No estudo foram incluídos 30 pacientes com pré-diagnóstico de SAOS por polissonografia. Para avaliação da função olfativa foi utilizado o teste de olfato Sniffin' Sticks antes da terapia com PAP e cerca de 3 meses após a terapia. Quatro pacientes receberam um dispositivo de pressão positiva contínua automática, 2 pacientes receberam dispositivo PAP de dois níveis e 24 pacientes receberam um dispositivo CPAP ${ }^{16}$.

Antes da terapia com PAP, foi detectada hiposmia em 21 pacientes e normosmia foi detectada em 9 pacientes. Depois da terapia, hiposmia foi detectada em 7 pacientes e normosmia foi detectada em 23 pacientes. Escores relativos a limiar de odor, descrição de odor e identificação de odor aumentaram significativamente após a terapia PAP ( $p=$ $0,001, p=0,002, p=0,001$, respectivamente). O escore limiar-descrição-identificação pós-tratamento $(32,50 \pm 3,88)$ foi significativamente maior que o pré-tratamento $(28,48$ $\pm 4,71)(p=0,001)^{16}$.

Dessa forma, o estudo coaduna com a possibilidade de que o tratamento da SAOS com uso de pressão positiva tem potencial de afetar positivamente nos sintomas de disfunção olfatória, entretanto seu " $n$ " pequeno e sua ausência de grupo controle fragilizam a validade externa do estudo ${ }^{16}$.

\section{Uso da estimulação cerebral}

Identificamos um estudo que comparou o escore pré-operatório da terapia de estimulação cerebral profunda (ECP) com o primeiro e terceiros meses pós operatórios em pacientes com doença de Parkinson (DP). Neste, houve melhora significativa para os testes de limiar do odor (LO) $(p<0,001)$, identificação do odor (IO) $(p<0,001)$ e o escore TDI $(p<0,001)$. Entretanto, se comparado o pré-operatório apenas com o 3 mês pósoperatório, além dos supracitados, também houve diferença $(p<0,001)$ no teste de discriminação do odor (DO) ${ }^{17}$. 
Manejo terapêutico do paciente com disfunção olfatória: revisão da literatura Bedaque HP; et al

Dessa forma, por haver divergências na literatura e devido a algumas limitações no estudo, como o curto período de acompanhamento, são necessários estudos adicionais, mais robustos e bem desenhados metodologicamente, para avaliar os correlatos funcionais do tratamento com ECP subtalâmica em pacientes com DP ${ }^{17}$.

\section{Uso da solução citrato de sódio intranasal}

As últimas pesquisas colocaram em evidência a importância do cálcio no desempenho das cascatas de sinalização a jusante dos receptores olfatórios, tendo principalmente um feedback inibitório. O cálcio tem um papel inibitório na transdução do sinal nervoso, que envolve a interferência dependente de cálcio-calmodulina com canais bloqueados por nucleotídeos e fosforilação dependente de cálcio e, assim, inativação da adenilil ciclase. A redução da concentração de cálcio livre na camada de muco nasal pode impedir o influxo intracelular desse íon necessário para ação inibitória. Sendo assim, O citrato de sódio atuaria como um sequestrante de cálcio, levando a melhora da função olfativa ${ }^{18}$.

Nesse sentido, foi realizado um estudo prospectivo, placebo controlado, únicocego, com 57 pacientes com hiposmia, com idades entre 22 e 79 anos. As causas de hiposmia incluíram: pós-viral (7); pós-traumático (10); doença sinonasal (30) e idiopática (10). Os pacientes foram tratados com uma única aplicação de $1 \mathrm{ml}$ de solução intranasal de citrato de sódio (3,5g / 140ml, pH 7,4, osmolaridade 298) à cavidade nasal direita ou esquerda ${ }^{18}$. A cavidade nasal contralateral foi tratada com uma única aplicação de $1 \mathrm{ml}$ de solução fisiológica de cloreto de sódio (placebo). O lado do tratamento com citrato de sódio foi randomizado entre os pacientes ${ }^{18}$.

Como resultado, houve melhora significativa no escore de identificação apenas no subgrupo com hiposmia pós-viral em comparação ao placebo, após tratamento com citrato de sódio $(p=0,02)$. Entretanto não houve melhora significativa para o limiar olfatório geral ou na discriminação geral $(p=0,36$ e $p=0,97)$. Assim, o estudo levanta a possibilidade do efeito benéfico do citrato apenas quando a etiologia da disfunção olfatória for por pós-viral ${ }^{18}$.

Os dados e resultados dos artigos encontrados foram compilados no Quadro 1, conforme mostrado a seguir: 
Manejo terapêutico do paciente com disfunção olfatória: revisão da literatura

Bedaque HP; et al

Quadro 1 - Sinopse dos dados dos artigos avaliados sobre anosmia

\begin{tabular}{|c|c|c|c|c|c|}
\hline Título & Autor & Ano & Desenho & Terapêutica & Desfecho \\
\hline $\begin{array}{l}\text { Intranasal sodium citrate solution improves } \\
\text { olfaction in post-viral hyposmia }\end{array}$ & Whitcroft et al & 2016 & ECR & $\begin{array}{l}\text { Lavagem nasal com } \\
\text { citrato de sódio }\end{array}$ & $\begin{array}{l}\text { Benefício na identificação dos odores } \\
\text { apenas quando etiologia pós-viral }\end{array}$ \\
\hline $\begin{array}{l}\text { Effects of olfactory } \\
\text { training: a meta-analysis. Rhinology }\end{array}$ & Sorokowska et al & 2017 & Metanálise & $\begin{array}{l}\text { Treinamento } \\
\text { olfatório }\end{array}$ & $\begin{array}{l}\text { Efeito positivo para identificação, } \\
\text { discriminação e TDI SCORE }\end{array}$ \\
\hline $\begin{array}{l}\text { Pharmacologic treatment for postviral } \\
\text { olfactory } \\
\text { dysfunction: a systematic review }\end{array}$ & Harless e Liang & 2016 & $\begin{array}{l}\text { Revisão } \\
\text { Sistemática }\end{array}$ & $\begin{array}{l}\text { Tratamentos } \\
\text { farmacológicos }\end{array}$ & $\begin{array}{l}\text { Benefício encontrado para corticoide oral, } \\
\text { corticoide local, ácido alfalipóico e } \\
\text { caroverinas. }\end{array}$ \\
\hline $\begin{array}{l}\text { Effect of Intranasal Insulin on Olfactory } \\
\text { Recovery in Patients } \\
\text { with Hyposmia: A Randomized Clinical Trial }\end{array}$ & Rezaeian & 2018 & ECR & Insulina Intranasal & $\begin{array}{l}\text { Benefício para o grupo com insulina } \\
\text { intranasal. }\end{array}$ \\
\hline Medical treatment of traumatic anosmia & Jiang et al & 2015 & ECR & Zinco e Corticoide & $\begin{array}{l}\text { Benefício para os grupos tratados com } \\
\text { Zinco e/ou corticoide }\end{array}$ \\
\hline $\begin{array}{l}\text { Acquired Olfactory } \\
\text { Dysfunction in Children and Adolescents: A } \\
\text { Systematic Review of the Literature }\end{array}$ & Schriever et al & 2018 & $\begin{array}{l}\text { Revisão } \\
\text { Sistemática }\end{array}$ & $\begin{array}{l}\text { Treinamento } \\
\text { olfatório }\end{array}$ & Benefício para o treinamento olfatório \\
\hline $\begin{array}{l}\text { Therapeutic use of steroids in non-chronic } \\
\text { rhinosinusitis olfactory dysfunction: a } \\
\text { systematic evidence-based review with } \\
\text { recommendations }\end{array}$ & Yan et al & 2019 & $\begin{array}{l}\text { Revisão } \\
\text { Sistemática }\end{array}$ & Corticoide & Benefício para o uso do corticoide oral \\
\hline $\begin{array}{l}\text { Budesonide irrigation with olfactory } \\
\text { training improves } \\
\text { outcomes compared with olfactory training } \\
\text { alone in patients with olfactory loss }\end{array}$ & Nguyen e Patel & 2018 & ECR & Corticoide nasal & $\begin{array}{l}\text { Adição de corticoide nasal melhorou a } \\
\text { resposta de pacientes fazendo } \\
\text { treinamento olfatório }\end{array}$ \\
\hline $\begin{array}{l}\text { The therapeutic effect of deep brain } \\
\text { stimulation on olfactory functions and } \\
\text { clinical scores in Parkinson's disease }\end{array}$ & Saatçi et al & 2019 & EC & $\begin{array}{l}\text { Estimulação } \\
\text { Cerebral Profunda }\end{array}$ & $\begin{array}{l}\text { Em pacientes com doença de Parkinson a } \\
\text { intervenção trouxe benefício para função } \\
\text { olfatória }\end{array}$ \\
\hline
\end{tabular}

Continua... 
Manejo terapêutico do paciente com disfunção olfatória: revisão da literatura

Bedaque HP; et al

\begin{tabular}{|c|c|c|c|c|c|}
\hline Título & Autor & Ano & Desenho & Terapêutica & Desfecho \\
\hline $\begin{array}{l}\text { Comparison of the } \\
\text { effects of nasal steroids and montelukast } \\
\text { on olfactory functions in patients } \\
\text { with allergic rhinitis }\end{array}$ & Dalgic et al & 2017 & ECR & $\begin{array}{l}\text { Corticoide nasal } \\
\text { e/ou Montelucaste }\end{array}$ & Benefício para o grupo do corticoide nasal \\
\hline $\begin{array}{l}\text { Does positive airway } \\
\text { pressure therapy improve olfactory } \\
\text { function? }\end{array}$ & Koseoğlu et al & 2017 & $\mathrm{EC}$ & $\begin{array}{l}\text { Pressão positiva } \\
\text { nas vias aéreas }\end{array}$ & $\begin{array}{l}\text { Em pacientes com SAOS houve benefício } \\
\text { no olfato com uso da pressão positiva }\end{array}$ \\
\hline $\begin{array}{l}\text { Efficacy of olfactory training in patients } \\
\text { with olfactory loss: a systematic review and } \\
\text { meta-analysis }\end{array}$ & Pekala et al & 2016 & Metalálise & $\begin{array}{l}\text { Treinamento } \\
\text { olfatório }\end{array}$ & $\begin{array}{l}\text { Benefício a favor do uso do treinamento } \\
\text { olfatório. }\end{array}$ \\
\hline $\begin{array}{l}\text { Olfaction in chronic } \\
\text { rhinosinusitis: comparing two different } \\
\text { endonasal steroid application methods }\end{array}$ & Poletti et al & 2017 & $\mathrm{EC}$ & $\begin{array}{l}\text { Corticoide spray ou } \\
\text { AMSA }^{\circledR}\end{array}$ & $\begin{array}{l}\text { Não houve diferença entre os tipos de } \\
\text { administração }\end{array}$ \\
\hline
\end{tabular}

ECR: Ensaio Clínico Randomizado; EC: Ensaio Clínico; SAOS: Síndrome da Apneia Obstrutiva do Sono; AMSA ${ }^{\circledR}$ : Dispositivo de inalação nasal por pressão pulsátil. 
Manejo terapêutico do paciente com disfunção olfatória: revisão da literatura

Bedaque HP; et al

\section{CONCLUSÃO}

A presente revisão da literatura buscou conhecer as publicações mais recentes no que tange o tratamento da disfunção olfatória. Um tema que certamente irá ganhar mais relevância diante do aumento desta sintomatologia em pacientes que tiveram COVID-19.

Em nossa análise identificamos o uso já recorrente do corticoide, nasal e/ou oral como parte integrante do tratamento para as diferentes etiologias da disfunção, bem como o uso do treinamento olfatório com bons e consistentes resultados, mesmo que a maioria dos estudos ainda sejam pequenos e com risco aumentado de vieses.

Ademais, 07 terapias com muitos poucos estudos científicos aparecem como oportunidades, são elas: o uso da insulina intranasal, a ventilação com pressão positiva nos pacientes com SAOS, a estimulação cerebral profunda nos pacientes com doença de Parkinson, a lavagem nasal com solução de citrato de sódio, Zinco, ácido alfalipóico e caroverinas. São poucos e frágeis, porém indicam a necessidade de novas pesquisas na área das alterações olfativas para buscar novas oportunidades de tratamento.

\section{REFERENCES}

1. Stevenson RJ. An initial evaluation of the functions of human olfaction. Chem Senses. 2010;35(1):3-20. doi:10.1093/chemse/bjp083.

2. Schriever VA, Gellrich J, von der Hagen M, Hummel T. Acquired Olfactory Dysfunction in Children and Adolescents: A Systematic Review of the Literature. Chem Senses. 2018 Sep 22;43(8):571-581. doi: 10.1093/chemse/bjy046. PMID: 30020456.

3. Tratado de Otorrinolaringologia / organização Shirley Shizue Nagata Pignatari, Wilma Terezinha Anselmo-Lima. - $3^{\circ}$ ed, - Rio de Janeiro: Grupo Editorial Nacional, 2020.

4. Lechien JR, Chiesa-Estomba CM, De Siati DR, et al. Olfactory and gustatory dysfunctions as a clinical presentation of mild-to-moderate forms of the coronavirus disease (COVID-19): a multicenter European study [published online ahead of print, 2020 Apr 6]. Eur Arch Otorhinolaryngol. 2020;10.1007/s00405-020-05965-1. doi:10.1007/s00405-020-05965-1

5. MENG, Xiangming et al. COVID-19 and anosmia: A review based on up-to-date knowledge. American Journal of Otolaryngology, p. 102581, 2020.

6. Harless L, Liang J. Pharmacologic treatment for postviral olfactory dysfunction: a systematic review. Int Forum Allergy Rhinol. 2016 Jul;6(7):760-7. doi: 10.1002/alr.21727. Epub 2016 Feb 16. PMID: 26879592.

7. Nguyen TP, Patel ZM. Budesonide irrigation with olfactory training improves outcomes compared with olfactory training alone in patients with olfactory loss. Int 
Manejo terapêutico do paciente com disfunção olfatória: revisão da literatura

Bedaque HP; et al

Forum Allergy Rhinol. 2018 Sep;8(9):977-981. doi: 10.1002/alr.22140. Epub 2018 Jun 14. PMID: 29901865.

8. Poletti SC, Batashev I, Reden J, Hummel T. Olfaction in chronic rhinosinusitis: comparing two different endonasal steroid application methods. Eur Arch Otorhinolaryngol. 2017 Mar;274(3):1431-1435. Doi: 10.1007/s00405-016-4341-6. Epub 2016 Oct 11. PMID: 27730325.

9. Dalgic A, Dinc ME, Ulusoy S, Dizdar D, Is A, Topak M. Comparison of the effects of nasal steroids and montelukast on olfactory functions in patients with allergic rhinitis. Eur Ann Otorhinolaryngol Head Neck Dis. 2017 Sep;134(4):213-216. doi: 10.1016/j.anorl.2016.05.012. Epub 2017 Jan 18. PMID: 28109729.

10. Yan $\mathrm{CH}$, Overdevest JB, Patel ZM. Therapeutic use of steroids in non-chronic rhinosinusitis olfactory dysfunction: a systematic evidence-based review with recommendations. Int Forum Allergy Rhinol. 2019 Feb;9(2):165-176. Doi: 10.1002/alr.22240. Epub 2018 Nov 24. PMID: 30472771.

11. Blomqvist EH, Lundblad L, Bergstedt H, Stjarne P. Placebo-controlled, randomized, double-blind study evaluating the efficacy of fluticasone propionate nasal spray for the treatment of patients with hyposmia/ anosmia. Acta Otolaryngol. 2003;123:862-868.

12. Pekala K, Chandra RK, Turner JH. Efficacy of olfactory training in patients with olfactory loss: a systematic review and meta-analysis. Int Forum Allergy Rhinol. 2016 Mar;6(3):299-307. doi: 10.1002/alr.21669. Epub 2015 Dec 1. PMID: 26624966; PMCID: PMC4783272.

13. Sorokowska A, Drechsler E, Karwowski M, Hummel T. Effects of olfactory training: a meta-analysis. Rhinology. 2017 Mar 1;55(1):17-26. doi:10.4193/Rhin16.195. PMID: 28040824.

14. Jiang RS, Twu CW, Liang KL. Medical treatment of traumatic anosmia. Otolaryngol Head Neck Surg. 2015 May;152(5):954-8. Doi: 10.1177/0194599815571272. Epub 2015 Feb 24. PMID: 25715353.

15. Rezaeian A. Effect of Intranasal Insulin on Olfactory Recovery in Patients with Hyposmia: A Randomized Clinical Trial. Otolaryngol Head Neck Surg. 2018 Jun;158(6):1134-1139. doi: 10.1177/0194599818764624. Epub 2018 Mar 20. PMID: 29557250.

16. Koseoğlu S, Derin S, Yilmaz M, Kutlu G, Sahan M. Does positive airway pressure therapy improve olfactory function? Int Forum Allergy Rhinol. 2017 Jun;7(6):557560. doi: 10.1002/alr.21923. Epub 2017 Mar 8. PMID: 28272841.

17. Saatçi Ö, Yılmaz NH, Zırh A, Yulug B. The therapeutic effect of deep brain stimulation on olfactory functions and clinical scores in Parkinson's disease. J Clin Neurosci. 2019 Oct;68:55-61. doi: 10.1016/j.jocn.2019.07.055. Epub 2019 Aug 2. PMID: 31383472.

18. Whitcroft KL, Merkonidis C, Cuevas M, Haehner A, Philpott C, Hummel T. Intranasal sodium citrate solution improves olfaction in post-viral hyposmia. Rhinology. 2016 Dec 1;54(4):368-374. doi: 10.4193/Rhin16.054. PMID: 27316224. 